\title{
Fairness of MAC Protocols: IEEE 1901 vs. 802.11
}

\author{
Christina Vlachou \\ EPFL, Lausanne, Switzerland \\ christina.vlachou@epfl.ch
}

\author{
Julien Herzen \\ EPFL, Lausanne, Switzerland \\ julien.herzen@epfl.ch
}

\author{
Patrick Thiran \\ EPFL, Lausanne, Switzerland \\ patrick.thiran@epfl.ch
}

\begin{abstract}
The MAC layer of the IEEE 1901 standard for power line communications employs a CSMA/CA method similar to, but more complex than, this of IEEE 802.11 for wireless communications. The differences between these protocols raise questions such as which one performs better and under what conditions.

We study the fairness of the 1901 MAC protocol in single contention domain networks, where all stations hear each other. We examine fairness at the packet level: a MAC layer protocol is fair if all stations share equitably the medium during a fixed time interval. We focus on short-term fairness, that is, over short time intervals. Short-term fairness directly impacts end-user experience, because unfair protocols are susceptible to introduce substantial packet delays. We evaluate short-term fairness with two metrics: Jain's fairness index and the number of inter-transmissions. We present test-bed results of both protocols and compare them with simulations. Both simulation and testbed results indicate that 802.11 is fairer in the short-term when the number of stations $N$ is between 2 and 5 . However, simulation results reveal that 1901 is fairer in the short-term for $N \geq 15$. Importantly, our test-bed measurements indicate that 1901 unfairness can cause significant additional delay when $N=2$. Finally, we confirm these results by showing analytically that 1901 is short-term unfair for $N=2$.
\end{abstract}

Index Terms-HomePlug, Medium Access Control (MAC), CSMA/CA, fairness, inter-transmissions, Jain's fairness index.

\section{INTRODUCTION}

Power line communications are one of the fastest developing technologies in home networking. The MAC layer of IEEE 1901 [5] (also known as HomePlug AV) for high data rate power line communications uses a carrier sense multiple access with collision avoidance (CSMA/CA) method. CSMA/CA protocols aim at minimizing the probability of collision, i.e. that two or more stations transmit simultaneously. To avoid this, the stations wait for a random interval $B C$, called the backoff counter, before each packet transmission. $B C$ is chosen uniformly at random between 0 and $C W-$ 1 , where $C W$ is the contention window. Previous studies e.g. [12], [6], have shown that CSMA/CA mechanisms affect the fairness of the system. In contrast to the very large body of research on the IEEE 802.11 CSMA/CA mechanism, which we briefly review in Section II, the fairness of the IEEE 1901 protocol has not yet been investigated ${ }^{1}$. In comparison with 802.11, 1901 is much more complex and details accounting for this complexity remain unexplained to a large extent. This intricacy compounds the design of analytical models and is

\footnotetext{
${ }^{1}$ From now on we shall refer to the IEEE 802.11 MAC as 802.11 and to the IEEE 1901 MAC as 1901.
}

the motivation of our work. We will use two different metrics, described in Section III, for evaluating short-term fairness, first by simulation and testbed results in Section IV, and next analytically for 2 stations in Section V.

Fairness is achieved when the medium is shared equitably among the stations during a fixed time interval. Depending on the length of this interval, fairness is defined as long-term or as short-term, and determines the quality of service. Longterm fairness is related to the average throughput, and shortterm fairness affects instantaneous delay. In an unfair system, a station has to wait for many other stations to transmit before it transmits again, which increases delay. We examine short-term fairness for two reasons. First, short-term fairness is a stronger property than long-term fairness: short-term fairness implies long-term fairness, but the reverse does not hold. Second, in the long-term, by the law of large numbers, all stations share equitably the medium, when they all have the same channel conditions, and there is no hidden terminal (as we assume in this paper).

Time division multiple access (TDMA) is the optimal method to share the medium in terms of short-term fairness. 1901 supports TDMA [5], but the default access method used in practice is CSMA/CA, as observed from commercial devices (devolo AVeasy). The 1901 CSMA/CA method uses 4 priority levels CA0-CA3 (from the lowest to the highest), but only the highest priority stations contend for the channel. By default, the priority of the packets is CA1. When all stations use the same priority level, CA0 and CA1 are the least fair, due to the difference in the $C W$ sizes across the levels (see Section III-B). In fact, the stations experience large delays because of the default settings of 1901, as shown in Section IV.

The main difference between 802.11 and 1901 stems from the factor causing a doubling of $C W$. In 802.11, if a station detects a collision after its transmission, it doubles $C W$ and selects a new $B C$. This is the only case where $C W$ is doubled. Whereas, with 1901, $C W$ is doubled not only after a collision, but it can also be doubled after sensing the medium busy, as explained in Section III-B. We observe that this difference strongly affects short-term fairness.

In a short-term fair system, after a station transmits, the other stations should a have higher probability of accessing the channel. The maximum value of $B C$ affects fairness. $B C$ is decremented by 1 at each time slot during which the medium is sensed idle. When stations sense the medium busy they pause $B C$, and resume it when the medium is idle again. Assume that all stations choose $B C$ uniformly at random over 
the range $[0, C W-1]$. In 802.11 , if a station transmits, then after the transmission this station has the lowest probability of transmitting again, because the other stations have a $B C$ in a smaller range (they already have counted some idle time slots and they resume their backoff). Whereas, in 1901 the stations sense the medium busy and select a new $B C$ over a larger range ( $C W$ is doubled), which is a source of unfairness as we will see in Section IV.

\section{RELATED WORK}

A large number of analytical models evaluating throughput, delay and fairness were proposed for 802.11 (e.g. [6]-[8], [12]). In particular, the model proposed by Bianchi [7] for 802.11 in single contention domain networks under saturated assumptions is very popular.

The CSMA/CA mechanism of 1901 is the same for the HomePlug 1.0, HomePlug AV, and HomePlug Green PHY specifications [2]. Chung et al. [9] proposed a model of the HomePlug 1.0 CSMA/CA mechanism, similar to Bianchi's. The complexity of 1901 is revealed by comparing the two models. First, both models are Markov chains, but the 802.11 model requires a 2-dimensional state space, whereas 1901 requires a 3-dimensional state space. Second, Bianchi computes the collision probability, but Chung et al. have to introduce also the probability of sensing the medium busy, because stations change differently their state in this case. Third, to compute these probabilities, [9] solves numerically a system of more than 1000 non-linear equations, and it is impossible to derive a fixed-point equation as in [7]. Although Chung et al. investigate delay, they do not examine short-term fairness related to the delay a packet experiences.

We explain the differences between the two MAC protocols. A comparison of the two PHY layers is given in [13]. Metrics such as throughput and coverage are related to the PHY layer, whereas short-term fairness is related to the MAC layer.

\section{BACKGROUND}

\section{A. Metrics on Short-Term Fairness}

Short-term fairness is investigated for 802.11 in [6] and [8] for single domain networks. We analyze similarly the 1901 protocol and evaluate fairness using two metrics: Jain's fairness index and the number of inter-transmissions, as used in [6] for 802.11.

Jain's fairness index is used to measure fairness, because it represents the variability of a set of measurements (e.g. the throughput of the stations). Larger values of Jain's index indicate better fairness. Ideally, when all stations share equitably the channel, this index is equal to 1 .

Definition 1. Let $N$ be the number of stations in the network and $x_{i}$ be the throughput of station $i$ within a time period. Then Jain's fairness index [10] is defined as

$$
J=\frac{\left(\sum_{i=1}^{N} x_{i}\right)^{2}}{N \sum_{i=1}^{N} x_{i}^{2}} .
$$

We use the sliding window method (SWM) [12] to express fairness as a function of the time horizon. This method slides a window of size $w$ packets across a trace of successful transmissions. For each sequence of $w$ packets, Jain's fairness index is computed from (1), with $x_{i}$ being the fraction of transmissions performed by station $i$ within the window of $w$ packets. Jain's fairness index $J(w)$ associated with the window size $w$ is the average of the fairness values of all consecutive sequences of $w$ packets in the trace. As $w$ increases, the fairness time horizon increases. We use a normalized window $W$ with respect to $N$, given by $W=w / N$. We apply the SWM to traces of successfully received packets acquired from simulation and real-network tests in Section IV.

Although Jain's fairness index with the SWM is a good metric of short-term fairness, it is analytically intractable. We thus employ another metric called the number of intertransmissions introduced in [6] to analyze short-term fairness.

Definition 2.The number of inter-transmissions $K$ for a tagged station $B$ is the number of transmissions originating from other stations between two consecutive transmissions of $B$.

In other words, if $K=0$, after a successful transmission of $B$, the next transmission is done again by $B$. If $K=1$, $B$ transmits once, then another station transmits, and then $B$ transmits again. The ideal value of $K$ is $N-1$ if there are $N$ stations contending for the channel. In reality, $K$ is a random variable, and $\mathbb{E}[K]=N-1$ corresponds to an ideally fair protocol. The analysis of the distribution of $K$ for networks with 2 stations is presented in Section V.

\section{B. Similarities and Differences between 802.11 and 1901}

Both the 802.11 [4] and the 1901 [5] CSMA/CA mechanisms use a time-slotted random backoff procedure with a backoff counter $(B C)$ and a contention window $(C W)$. Fairness depends only on the backoff procedure, as after each transmission, the station with the minimum $B C$ transmits in the next transmission opportunity. In addition to $B C$ and $C W$, 1901 includes two more variables that are not used in 802.11: the backoff procedure counter $(B P C)$ and the deferral counter $(D C)$. The two backoff procedures are described by the finite state machine in Figure 1.

First, we discuss the similarities between the two methods. At each transmission attempt, the station starts at state Init and selects $B C$ uniformly at random over the range $\left[0, C W_{\min }-\right.$ 1], where $C W_{\min }$ denotes the minimum contention window (equal to 16 for $802.11 \mathrm{a}$ and to 8 for 1901). Then, the station moves to state Idle and decrements $B C$ by 1 at each time slot the medium is sensed idle. The station changes its state if $B C=0$ or if the medium is sensed busy.

If $B C=0$, the station moves to state $T x$ and transmits. After the transmission, the station moves to state Init. If the transmission is successful, the same procedure is executed for the next packet. If the transmission fails, the station chooses a new $B C$ uniformly at random over the range $\left[0,2 C W_{\min }-1\right]$ and retransmits when $B C=0$ again. $C W$ is doubled 
(B)

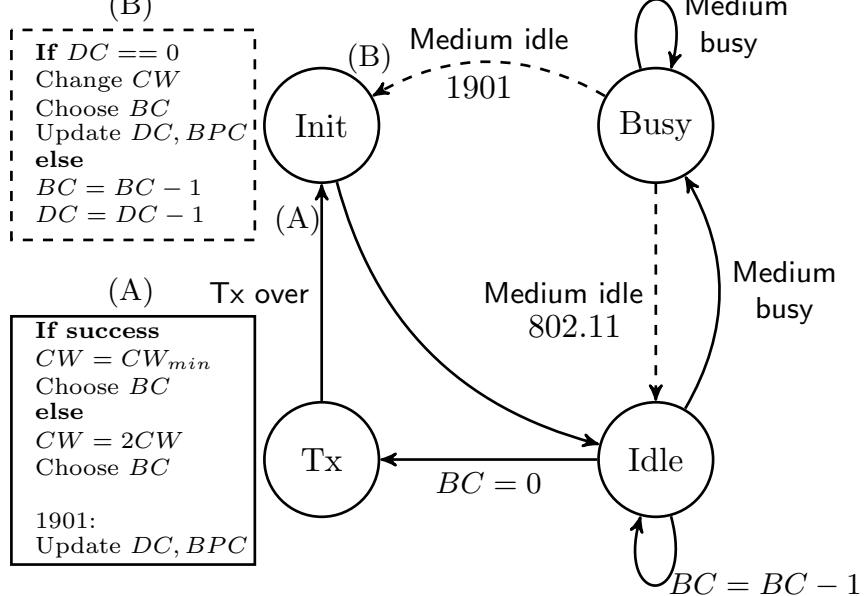

Fig. 1. A finite state machine representing the 802.11 and 1901 backoff procedures. A station is in state Init whenever it chooses $B C$, in states Busy and Idle when the medium is sensed busy and idle, respectively, and in state $T x$ when it is transmitting. After a transmission attempt, stations move to state Init and execute algorithm (A). In 1901 after choosing a new $B C$, the station updates $D C$ according to the old $B P C$ and then increases $B P C$ by 1. The dashed lines represent the only difference between the two standards. After sensing the medium idle while being in Busy state, 1901 stations move to state Init and execute algorithm (B). Note that the 1901 random backoff procedure performs the same as 802.11 if $D C \rightarrow \infty$ for all values of $B P C$.

\begin{tabular}{|c|c|c||c|c|}
\hline Priority: & \multicolumn{2}{|c||}{ CA0 and CA1 } & \multicolumn{2}{c|}{ CA2 and CA3 } \\
\hline$B P C$ & $C W$ & $D C$ & $C W$ & $D C$ \\
\hline \hline 0 & 8 & 0 & 8 & 0 \\
\hline 1 & 16 & 1 & 16 & 1 \\
\hline 2 & 32 & 3 & 16 & 3 \\
\hline$\geq 3$ & 64 & 15 & 32 & 15 \\
\hline
\end{tabular}

TABLE I

IEEE 1901 VALUES FOR THE CONTENTION WINDOW $C W$ AND THE DEFERRAL COUNTER $D C$. DIFFERENT PRIORITIES HAVE DIFFERENT $C W$.

whenever a transmission attempt fails, until a maximum value $C W_{\max }$ (equal to 1024 for 802.11a and to 64 for 1901).

We now move to the difference between the protocols. If the medium is sensed busy, then the station moves to the Busy state and does not change state until the medium is idle again. The mechanisms differ in the procedure the stations follow after the medium is idle again. In 802.11 the station returns to the Idle state, whereas in 1901 the station first moves to state Init. There, depending on the value of $D C$, the station either doubles its $C W$ and selects a new $B C$, or decrements $D C$ and $B C$ by 1 (Algorithm (B), Figure 1). $D C$ and $C W$ are chosen according to $B P C$ (Table I).

Let us briefly explain the rationale behind the complexity of 1901 and the new variables introduced by underlining three differences between the protocols. First, the $C W$ sizes are smaller for 1901 (the $1901 C W$ range is $8-64$, whereas for 802.11a the range is $16-1024)$. Given that the maximum length of a frame in 1901 is $2501.2 \mu \mathrm{s}$ and that the time slot duration is $35.84 \mu s$ [5], the $C W$ sizes of 1901 are small to minimize the time during which the channel is idle, and to maximize throughput. Second, $D C$ is introduced in 1901 to reduce the probability of collision, because small $C W$ sizes lead to high probability of collision. $D C$ obliges stations to double their $C W$ before they collide, that is after sensing a transmission in the channel, because at that point it is likely that many stations are waiting to transmit and have equal backoff counters. Although $D C$ achieves its goal, it causes short-term unfairness between the station that has transmitted successfully and the other stations that sense the medium busy, because the former has always the lowest $C W$, and therefore the highest probability of transmitting in the network. Third, $B P C$ in 1901 is the variable that keeps memory of the state at which the station is, so that the station is able to update $D C$ and $C W$. For instance, when CA3 stations have that $C W=16$ and need to update $D C$ and $C W$, in the absence of $B P C$ they would not be able to determine the new $D C$ and $C W$ values.

CA2-CA3 priorities have lower $C W$ sizes than CA0-CA1. This means that the collision probability is higher for CA2CA3, but the short-term fairness is better. There is a trade-off between collision probability and short-term fairness in 1901 . Larger $C W$ values cause lower collision probability and worse short-term fairness. Whereas, larger values of $D C$ result in higher collision probability and better short-term fairness.

In this paper we study and compare the aforementioned CSMA/CA mechanisms. Our assumptions for the simulation and the analysis are the following:

- Perfect sensing: The $N$ stations share a single contention domain (in particular, there is no hidden terminal).

- Perfect channel: No packet loss or error occurs because of the physical layer; transmission failures are due to collisions. The stations use the same physical data rate.

Furthermore, we compare the protocols under saturated conditions, where stations always have packets to transmit. This corresponds to conditions where the stations are capacityintensive, and fairness is thus a more challenging goal to achieve. We assume that all stations use the same priority.

\section{Simulation AND EXPERIMENTAL RESUlTS}

\section{A. Simulator Description and Experimental Methodology}

To compare the CSMA/CA mechanisms (802.11a and 1901), we developed a Matlab simulator. The simulator is time-slotted, features the backoff procedures of the two standards and assumes perfect sensing and error-free channel. We used the simulator to obtain a trace of successfully transmitted frames over the channel. We did not simulate the PHY layer, because the two channels are different, and the testbed experiments presented below are carried out on different mediums (power line and wireless).

In addition to our simulator, we built a test-bed of 6 stations, each comprising both a power line and a wireless communication interface. The stations are ALIX boards running the OpenWrt Linux distribution [3]. Each board is equipped with a Homeplug AV miniPCI card (Meconet interface, Intellon INT6300 chip), and an other miniPCI card for wireless communications (Atheros AR9220). We ran tests with both cards and first describe the methodology followed for the 1901 tests.

The Homeplug AV cards are powered from the miniPCI bus, which gives us the possibility of running tests isolated from the electrical grid. This setting is similar to [15] and 
reproduces the perfect channel assumption to overcome the PHY layer phenomena that might affect our measurements. We use coupling devices to connect the cards to a common wiring block. The stations are in a single contention domain and the perfect sensing assumption holds.

We employ Faifa [1] to obtain a trace of successfully transmitted 1901 packets. Faifa includes a sniffer mode with which we can retrieve the headers of the transmitted packets. The headers give information such as the source and the destination of the packet, the tone map used for the packet transmission, and the type of the packet (i.e. MPDU (MAC protocol data unit), beacon, ACK). We exploit this information to acquire the trace of successfully received MPDUs by a station during a fixed time interval of 2 minutes. During this time interval approximately $8 \cdot 10^{4}$ MPDUs were captured. We examine the fields of the headers of the captured packets to obtain the trace of received MPDUs. The field MPDU Count is equal to the number of the remaining MPDUs in the current burst transmission. MPDUs can belong to bursts and bursts contend for the medium, not individual MPDUs. Thus, we collect MPDUs with MPDU Count $=0$, as these MPDUs are either not part of a burst or are the last MPDUs of a burst [5].

In our setup, $N$ stations send UDP traffic to the $(N+1)$ th station using iperf. We run tests for $N=2,3,4,5$. The $(N+1)$ th station captures the MPDUs received using Faifa. In order for the saturated conditions to hold, we set the stations to send traffic at a rate higher than the link capacities. We performed tests at all priority levels. The priority of the packets was modified by changing the IP type of service (ToS) field with iperf, and we verified the priority change by examining the Link ID field of the MPDU header [5]. We demonstrate results for only CA1 priority used by default. CA2-CA3 priorities experience better short-term fairness. The results for priority CA0 are the same with those for CA1 and are not presented here.

We conduct the same tests with the wireless cards at $802.11 \mathrm{a}$ mode and channel 44 to avoid interference with other wireless networks. We employed tcpdump to capture packets. We chose Faifa for 1901, because tcpdump captures Ethernet packets and not the 1901 MPDUs due to frame aggregation.

\section{B. Results and Findings}

In this section we present our simulation and test-bed results. Our findings are developed in the following paragraphs.

1) The worst unfairness for 1901 occurs when $N=2$ :

Figure 2 shows the results of the SWM applied to traces of successfully transmitted packets from simulation and experiments for $N=2$. We observe that Jain's fairness index $J(W)$ is higher for 802.11a for all values of the normalized window $W$. Thus, 802.11 is fairer for all time horizons, and more particularly in the short-term. $J(W)=0.95$ is achieved with $W=5$ for 802.11a and with $W=70$ for 1901 .

2) As $N$ increases, 1901's fairness improves, but 802.11's fairness deteriorates. 1901 is short-term fairer than 802.11 for $N \geq 15$ : The short-term fairness of the protocols changes as the number of stations $N$ increases. Figures 3 and 4 show

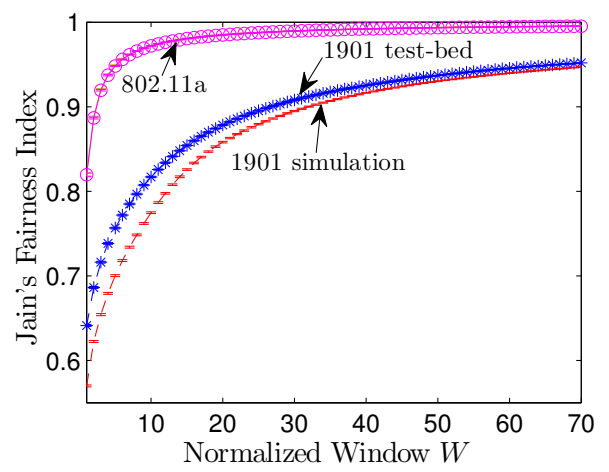

Fig. 2. Jain's fairness index with the SWM for $N=2$ from simulation and test-bed results. Simulation results coincide well with test-bed results.

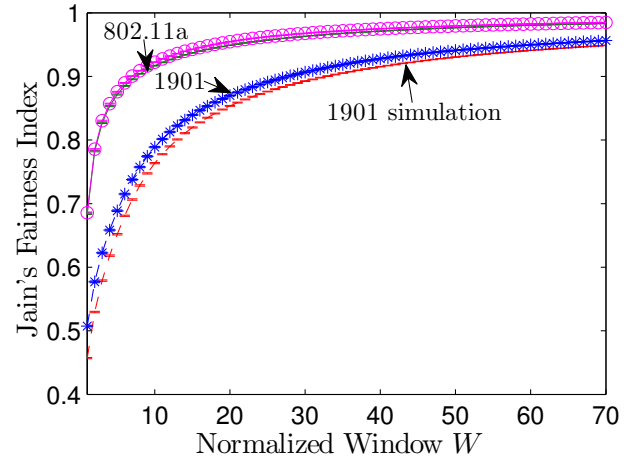

Fig. 3. Jain's fairness index with the SWM for $N=3$ from simulation and test-bed results. Simulation results coincide well with test-bed results.

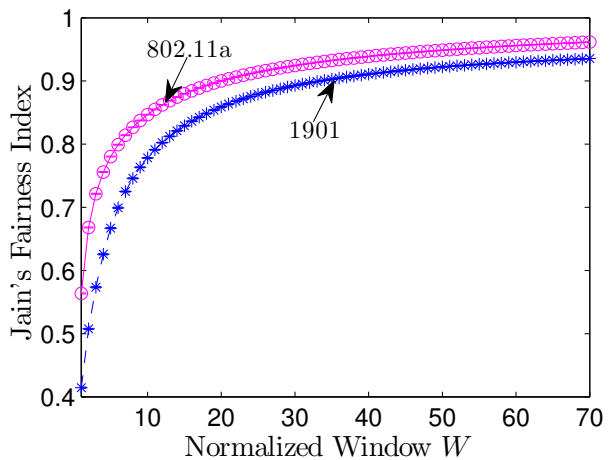

Fig. 4. Test-bed results of Jain's fairness index with the SWM for $N=5$.

the value of $J(W)$ from simulation and test-bed results for $N=3$ and 5 , respectively. The two curves for 1901 and 802.11a approach each other as $N$ increases (Figures 2, 3, 4). The largest difference between the mechanisms occurs for $N=2$, which is expected to be a common scenario in home networking. Figure 5 plots $J(W=1)$ as a function of $N$ in simulation. $J(W=1)$ for 802.11 a decreases as $N$ increases, which has already been observed in [6] and [8]. This is due to the collision probability that increases with $N$. In 802.11 for large $N$, after a collision, a station that transmitted successfully in the past is favored compared to the others, because there exists a subset of stations with a larger $C W$ due to collisions. Figure 5 also uncovers that 1901 is fairer in the short-term for $N \geq 15$. Furthermore, it illustrates two regimes that characterize the 1901 behavior; for $N<10, J(1)$ decreases, and for $N \geq 10, J(1)$ increases. When $N<10, J(1)$ for 1901 decreases for the same reason as for 802.11, as explained 
above. When $N \geq 10, J(1)$ increases because under high collision probability conditions, in 1901 there is not a subset of stations with low $C W$ contrary to 802.11: after a collision all stations (including those that transmitted successfully in the past) increase their $C W$ due to sensing the medium busy. Moreover, the increasing behavior of $J(1)$ for 1901 when $N$ is large is attributed to the high collision probability and to the inability of the maximum $C W\left(C W_{\max }=64\right)$ to accommodate collisions. Consequently, when $N$ is large, the majority of the stations have $C W=C W_{\max }$, hence they have the same probability of transmitting, which results in fairness.

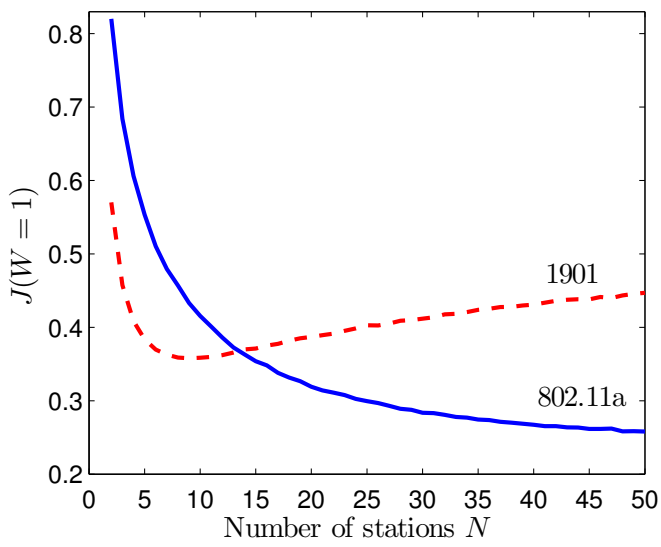

Fig. 5. $J(W=1)$ as a function of $N$ from simulation results. Short-term fairness improves for 1901 and deteriorates for 802.11 as $N$ increases.

3) 1901 short-term unfairness introduces substantial delay: Short-term unfairness increases delay in networks with a small number of stations. An example of this phenomenon with $N=2$ is presented in Figure 6, where a station $A$ sends UDP traffic with a varying load to a station $C$, while a station $B$ sends ping requests of size 1400 bytes to $C$. Figure 6 shows the median round trip time (RTT) of $10^{4}$ ping requests of $B$ for both 1901 (top) and 802.11a (bottom). The maximum RTT was $215.7 \mathrm{~ms}$ for 1901 and $11.5 \mathrm{~ms}$ for 802.11 when $A$ was saturated. The results reveal that in a scenario where $A$ sends saturated traffic and $B$ sends bursty traffic in the form of individual packets, $B$ experiences delays larger than the maximum acceptable delays of delay sensitive applications. The average and maximum RTT of $B$ when $A$ was saturated were 8.2 and $21 \mathrm{~ms}$, respectively, for CA3 priority.
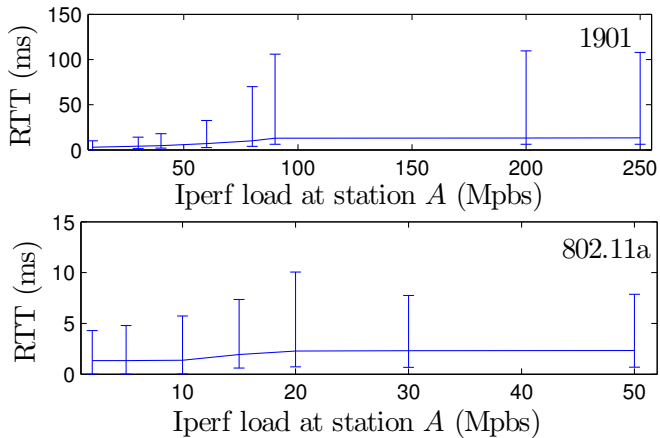

Fig. 6. The median RTT of $10^{4}$ ping requests of $B$ as a function of $A$ 's iperf load, measured from our test-bed. The error bars represent the $10 \%$ and $99 \%$ percentiles. Observe the difference in the scales of y-axes.
The difference between the test mentioned above and the saturated UDP traffic tests is that in the ping tests both stations have their $C W$ equal to $C W_{\min }$ when they start contending for the channel. In the next section, we analyze the distribution of inter-transmissions of station $B$ under this scenario, i.e. when $A$ and $B$ contend with their initial $C W$ equal to $C W_{\min }$.

\section{THE DISTRIBUTION OF INTER-TRANSMISSIONS}

We observed from simulation and experiments that the worst unfairness for 1901 occurs for $N=2$. Therefore, we examine this scenario further in this section, using analytical tools. We explore short-term fairness by computing the distribution of the number of inter-transmissions $K$.

We assume in this section that the backoff counters of the stations are continuous random variables uniformly distributed over the range $[0, C W]$. Hence, the probability of collision is 0 . This assumption is essential because the analytical model of the real discrete time protocol appears to be intractable. The computation of the distribution is based on the observation that the station with the smallest backoff counter transmits whenever the stations choose or resume their backoff counters.

Let $A$ and $B$ be the two stations. We tag station $B$. Let $b$ be the backoff counter of $B$ and $a_{i}$ be the backoff counter of the $A$ before the $i$ th transmission. Then, the distribution of $K$ for 802.11 is is given by

$$
\mathbb{P}(K=k)=\mathbb{P}\left(\sum_{i=1}^{k} a_{i}<b \text { and } \sum_{i=1}^{k+1} a_{i} \geq b\right) .
$$

For 802.11 , the distribution of $K$ is given by [6] and reads

$$
\mathbb{P}_{802.11}(K=k)=\frac{k+1}{(k+2) !}, k \geq 0 .
$$

The computation of this distribution for 1901 is more complex. Let $B C_{B}, C W_{B}$ and $D C_{B}$ be the values of $B C, C W$ and $D C$ at $B$, respectively. We define similarly the counters of $A$. In 802.11, under the collision-free assumption, the contention window remains constant. Whereas, in $1901 C W_{B}$ is doubled whenever $A$ transmits and $D C_{B}=0$, because $B$ senses the medium busy. To evaluate $\mathbb{P}_{1901}(K=k)$, we compute the probability that $A$ transmits $k$ times consecutively, or equivalently the probability that $B C_{B}<B C_{A}$ at each of the $k$ transmission attempts. $B C_{A}$ is always uniformly distributed over the range $[0,8]$, whereas $B C_{B}$ depends on k. $C W_{B}=8$ and $D C_{B}=0$ when $K=0 . B C_{B}$ is updated after each transmission of $A$ as follows: if $D C_{B}=0$, new $B C_{B}$ and $D C_{B}$ are chosen; otherwise, $B C_{B}$ is decremented by $B C_{A}$ and $D C_{B}$ is decremented by 1 . Using Table I, we compute $D C_{B}$ and the range to which $B C_{B}$ belongs for each $k$. The 1901 distribution of $K$ is given by (3), whose proof can be found in [14].

Proposition 1. The distribution of the number of intertransmissions $K$ for 1901 is

$$
\mathbb{P}_{1901}(K=k)= \begin{cases}1 / 2 & k=0 \\ 1 / 8 & k=1,2 \\ 1 / 32 & 3 \leq k \leq 6 \\ 0.0578^{l} / 128 & 7 \leq k-16 l \leq 14 \\ 0.0578^{l} \cdot \mathbf{I}(k-16 l-14) / 8 & 15 \leq k-16 l \leq 22,\end{cases}
$$


where $l=\lfloor(k-7) / 16\rfloor$, and $\mathbf{I}(n)$ is the nth entry $(1 \leq n \leq 8)$ of the vector $\mathbf{I}=10^{-2}(6.256 .256 .246 .175 .925 .384 .53$ 3.49).

An important consequence of Proposition 1 is that the distribution of $K$ has a heavier tail for 1901 than for 802.11, meaning that with 1901 a station is more likely to perform many more consecutive back-to-back transmissions than with 802.11. To see this, consider the survival functions of the 1901 and 802.11 distributions of $K, S_{1901}(k)=\mathbb{P}_{1901}(K>k)$ and $S_{802.11}(k)=\mathbb{P}_{802.11}(K>k)$. One can compute from (2) and (3) that

$$
\lim _{k \rightarrow \infty} \frac{S_{1901}(k)}{S_{802.11}(k)}=\infty,
$$

which means that the distribution of $K$ has a heavier tail for 1901 than for 802.11 [11]. This is also observed from the two distributions plotted in Figure 7, and from the moments of the distributions: $\mathbb{E}[K]=2.8$ and $\operatorname{Var}(K)=28.28$ for 1901 , and $\mathbb{E}[K]=0.73$ and $\operatorname{Var}(K)=0.77$ for 802.11 .
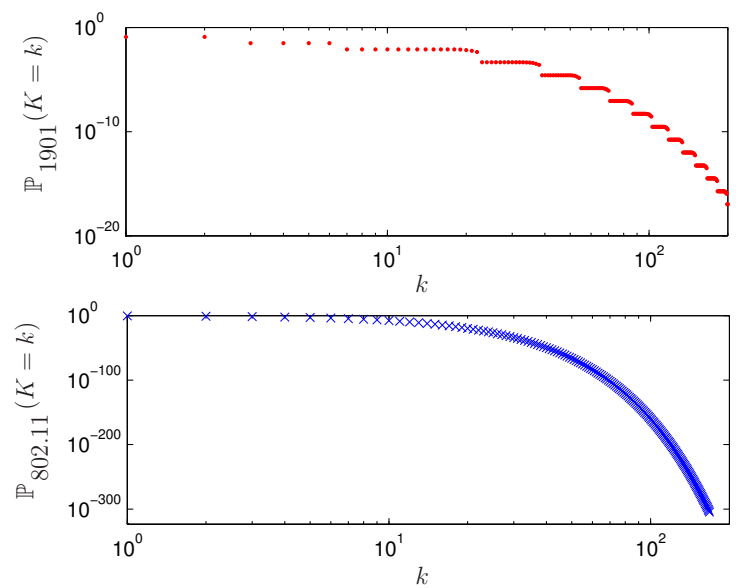

Fig. 7. The distribution of $K$ for 1901 (CA1 priority) and 802.11 computed analytically in $\log -\log$ scale. Note the difference of scale between the two standards, e.g. $\mathbb{P}_{1901}(K=15) \sim 10^{-3}$ and $\mathbb{P}_{802.11}(K=15) \sim 10^{-14}$. The 1901 distribution of $K$ attenuates periodically, as observed from (3)

The distribution of $K$ remains the same when we multiply all $C W$ values with the same constant. We ran our simulations with increasing $C W$ values, multiplying the $C W$ values by 2 . When $C W_{\min }=8$, we find that $\mathbb{E}[K]=5.5$ from simulations of 1901 . As $C W_{\min } \rightarrow \infty$, the probability of collision tends to 0 and the discrete distribution of the backoff counters can be approximated by the continuous one. With $C W_{\min }=1024$, the distribution of $K$ from the simulation of the real 1901 protocol coincides with the analytical distribution. This shows that our model is a good approximation of the real protocol and that collisions deteriorate short-term fairness for $N=2$, since $\mathbb{E}[K]=5.5$, when $C W_{\min }=8$.

\section{CONCLUSION AND Discussion}

We have studied the fairness characteristics of 1901 and given a comparison with 802.11. 802.11 is conservative and employs large $C W$ sizes to avoid collisions, whereas 1901 is more aggressive: it tries a small $C W$ first, but later engages a complex mechanism $(D C, B P C)$ to counter the high collision rate and to increase throughput. This extra complexity of 1901 comes at the price of fairness and delay. We have identified two regimes. When the number of stations is $N \leq 15,802.11$ is fairer than 1901. For $N \geq 15$ - a scenario that seems somewhat less relevant for home networking - 1901 is fairer. We have showed analytically that 1901 is significantly less fair than 802.11 when $N=2$. Our findings imply that 1901 can be detrimental to delay-sensitive traffic, such as voice or video, especially when the default settings are used with priority CA1. This suggests that end-user experience heavily relies on the existence of proper traffic classification mechanisms that assign priorities to traffic flows judiciously.

HomePlug Green PHY technology, which uses the same CSMA/CA mechanism as 1901, for smart grid applications will be integrated into the smart home. Our study suggests that the MAC protocol should be carefully designed to ensure the smooth operation of Green PHY applications when HomePlug AV stations send bursty traffic. Finally, our study of fairness at different timescales, along with its impact on delay, can be useful to help design protocols for hybrid wireless/power-line networks.

\section{ACKNOWLEDGMENT}

We would like to thank David Malone for his guidance regarding the experimental settings of our test-bed. This work is financially supported by a grant of the SmartWorld project of the Hasler Foundation, Bern, Switzerland.

\section{REFERENCES}

[1] Faifa. https://launchpad.net/ubuntu/+source/faifa

[2] HomePlug Alliance. http://www.homeplug.org/.

[3] OpenWrt. http://https://openwrt.org/.

[4] "IEEE Std 802.11-2007," pp. C1-1184, 122007.

[5] "IEEE Standard for Broadband over Power Line Networks: Medium Access Control and Physical Layer Specifications," pp. 1-1586, 302010.

[6] G. Berger-Sabbatel, A. Duda, O. Gaudoin, M. Heusse, and F. Rousseau, "Fairness and its impact on delay in 802.11 networks," in IEEE GLOBECOM '04, vol. 5, nov.-3 dec. 2004, pp. 2967-2973 Vol.5.

[7] G. Bianchi, "Performance analysis of the IEEE 802.11 distributed coordination function," Selected Areas in Communications, IEEE Journal on, vol. 18, no. 3, pp. 535-547, 2000.

[8] M. Bredel and M. Fidler, "Understanding Fairness and its Impact on Quality of Service in IEEE 802.11," in INFOCOM 2009, IEEE, pp. $1098-1106$

[9] M. Chung, M. Jung, T. Lee, and Y. Lee, "Performance analysis of HomePlug 1.0 MAC with CSMA/CA," Selected Areas in Communications, IEEE Journal on, vol. 24, no. 7, pp. 1411-1420, 2006.

[10] H. W. Jain R., Chiu D.M., "A quantitative measure of fairness and discrimination for resource allocation in shared computer systems," $D E C$ Research Report TR-301, 1984.

[11] S. Klugman, H. Panjer, G. Willmot, and G. Venter, Loss models: from data to decisions. Wiley New York, 1998, vol. 2, ch. 4.3.2.

[12] C. Koksal, H. Kassab, and H. Balakrishnan, "An analysis of shortterm fairness in wireless media access protocols (poster session)," $A C M$ SIGMETRICS Performance Evaluation Review, vol. 28, no. 1, pp. 118119, 2000.

[13] Y. Lin, H. Latchman, R. Newman, and S. Katar, "A comparative performance study of wireless and power line networks," Communications Magazine, IEEE, vol. 41, no. 4, pp. 54-63, 2003.

[14] C. Vlachou, J. Herzen, and P. Thiran. (2012) Inter-transmission distribution for IEEE 1901. https://documents.epfl.ch/users/v/vl/vlachou/www/ 1901_plc/inter_transmissions_1901.pdf.

[15] B. Zarikoff and D. Malone, "Construction of a plc test bed for network and transport layer experiments," in IEEE ISPLC, 2011, pp. $135-140$. 